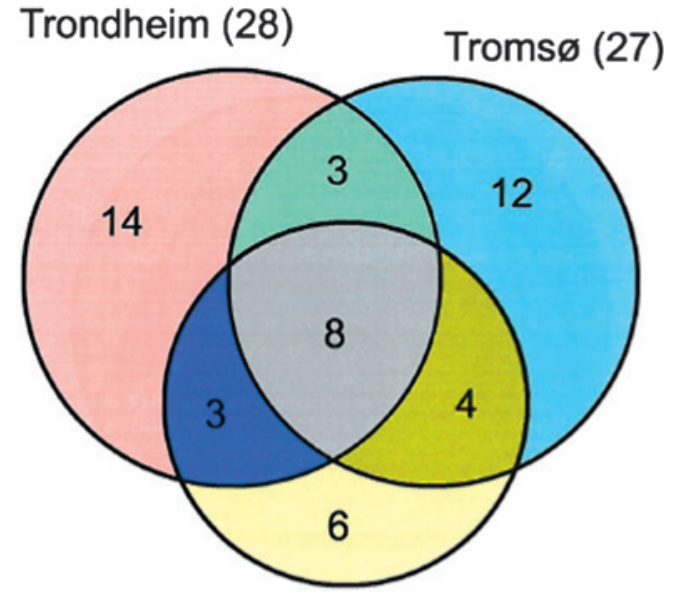

Finnmark (21)

Abstract P4-S1.06 Figure 1 Distribution of sequence types $(n=50)$ of $248 C$ trachomatis cases in the counties of Finnmark $(n=80)$, Troms $\emptyset$ $(\mathrm{n}=80)$, and Trondheim $(\mathrm{n}=88)$.

differences were not statistically significant.The common ompA genovar E predominated in Tromsø and Trondheim (55\%) whereas genovar G $(36 \%)$ was most frequent in Finnmark.

Conclusions Our MLST provided a significantly higher differentiation capacity than conventional ompA typing, which has predominated genotyping of $C$ trachomatis for decades. Thus highresolution MLST is a useful tool for molecular epidemiology of chlamydial infections.

\section{P4-S1.07 CHLAMYDIA TRACHOMATIS DNA STABILITY INDEPENDENT OF PRESERVATION TEMPERATURE, TYPE OF MEDIUM EN STORAGE DURATION}

doi:10.1136/sextrans-2011-050108.509

${ }^{1} \mathrm{~L}$ van Dommelen, ${ }^{2} \mathrm{~F} \mathrm{H}$ van Tiel, ${ }^{3} \mathrm{~N}$ H Dukers, ${ }^{2} \mathrm{P} F$ Wolffs, ${ }^{2} \mathrm{~S} B$ Herngreen, ${ }^{3} \mathrm{C}$ J P A Hoebe. ${ }^{1}$ PAMM, Veldhoven, Netherlands; ${ }^{2}$ Maastricht University Medical Centre, Netherlands; ${ }^{3}$ South Limburg Public Health Service, Netherlands

Background In almost all diagnostic studies, stored samples are used. This is also the case in Chlamydia trachomatis research. The performance of diagnostic assays, such as nucleic acid amplification tests, is dependent on the preservation of $C$ trachomatis DNA. However, the influence of different storage conditions on $C$ trachomatis DNA has not been thoroughly explored yet. Therefore, we have studied the impact of temperature, type of medium and duration of storage on the preservation of $C$ trachomatis DNA.

Methods Phosphate buffered saline (PBS), 2-sucrose-phosphate medium (2-SP), COBAS Amplicor medium and urine samples were spiked with $C$ trachomatis serovar D elementary bodies and stored at room temperature, $4^{\circ} \mathrm{C},-20^{\circ} \mathrm{C}$ and $-80^{\circ} \mathrm{C}$. DNA was isolated using the Qiagen DNA mini kit (Qiagen $\mathrm{GmbH}$, Hilden, Germany). Samples were tested in triplicate with a TaqMan PCR at day 0, 1, 7, 14 and 30. Furthermore, clinical $C$ trachomatis positive urine samples were collected and pooled, and stored and tested in triplicate, at the same temperatures and time intervals. This same procedure was executed with pooled $C$ trachomatis positive swab samples.

Results $C$ trachomatis was detected in all samples, irrespective of the different storage temperature conditions, different types of medium and the different durations of storage. Regarding storage time, the cycle threshold (ct) overall did not increase, in fact it tended to decrease within 30 days. If there was an increase in ct over time, this occurred in $<10 \%$ of the experiments, it did not outreach three (corresponding to a decline in load of $1 \mathrm{log}$ ). This mainly occurred in the spiked COBAS samples which were frozen.

Conclusions All samples tested positive for $C$ trachomatis in our experiments, with on average no decrease in cycle threshold over time. We can therefore conclude that PBS, 2-SP, COBAS Amplicor medium and urine are all excellent media to preserve $C$ trachomatis DNA for a longer period of time, independent of storage temperature.

\section{P4-S1.08 PERFORMANCE COMPARISON OF NEISSERIA GONORRHOEAE (NG) SEQUENCE TYPING METHODS NG- MAST AND NG-MLVA ON A DEFINED PANEL OF ISOLATES}

doi:10.1136/sextrans-2011-050108.510

${ }^{1} \mathrm{R}$ Heymans, ${ }^{2} \mathrm{D}$ Golparian, ${ }^{1} \mathrm{~S}$ Bruisten, ${ }^{2} \mathrm{M}$ Unemo. ${ }^{1}$ Health Service of Amsterdam (GGD), Amsterdam, Netherlands; ${ }^{2}$ Örebro University Hospital, Sweden

Background The increase in $N$ gonorrhoeae (NG) prevalence since the mid-1990s, increasing antibiotic resistance, and the absence of an effective vaccine, demand more efficient national and international surveillance of the NG epidemiology. Accordingly, objective, robust and discriminatory genotyping is a crucial tool. The performance characteristics of the internationally frequently used NG multiantigen sequence typing (NG-MAST) and the recently developed multi-locus variable-number tandem repeat analysis (NG-MLVA), were compared.

Methods From 2002 to 2003, NG isolates ( $n=330$ ) were obtained from multiple anatomical locations from the same patient $(n=118)$ and from self reported sexual contacts (54 couples). These isolates, were genotyped using NG-MAST, by sequencing of more polymorphic segments of porB and tbpB, and by NG-MLVA, which examines polymorphisms in five different tandem repeat loci.

Results From the 118 patients with multiple isolates per visit, 97 $(82.2 \%)$ had identical NG-MAST STs. Of these 97, 68 patients (57.6\%) had isolates with identical MLVA types, 22 patients $(18.6 \%)$ had single-locus variants (SLVs), six patients (5.1\%) had doublelocus variants (DLVs), and one patient $(0.8 \%)$ had a multilocus variant. Of the isolates of 13 patients $(11.0 \%)$ with non-identical NG-MAST STs, nine patients had identical MLVA types, three patients had SLVs, and one patient had a DLV. For the isolates of the remaining eight patients (6.8\%), both typing methods identified divergent genotypes. So for 76 patients (64\%) fully concordant clustering was obtained. Considering a SLV as an identical MLVA type the concordance between the two techniques increased to $83 \%$, From 44 of the 54 couples $(81.5 \%)$, the isolates of the patient and the contact had identical NG-MAST STs, while there were 34 MLVA couples $(63.0 \%)$ with identical MLVA types, seven couples $(13.0 \%)$ with SLVs, and three couples $(5.6 \%)$ with DLVs. The isolates of 6 couples $(11.1 \%$ ) had identical MLVA types with different NG-MAST STs. In the isolates of four couples (7.4\%) both typing methods identified divergent genotypes. So for 45 couples (83\%) the two typing techniques were concordant.

Conclusions For efficient international surveillance of gonorrhoea transmission, objective, highly discriminatory, but stable genotyping is essential. Both NG-MAST and NG-MLVA displayed a high resolution and are sequence-based portable genotyping methods. They can either be used singly or in combination, depending on epidemiological questions. 